\title{
Estratégia de Saúde da Família no bairro Jardim Surubi/Itaperuna(RJ): análise de Acompanhamentos Hipertensão Arterial e Diabetes
}

Family Health Strategy in the Jardim Surubi / Itaperuna neighborhood (RJ): analysis of Acompanhamentos Hypertensão e Diabetes

De la salud de la salud en el Jardín Surubi / Itaperuna (RJ): analysis of Acompañamientos Hipertensión y Diabetes

\author{
Amanda Vargas PEREIRA (1) \\ Amanda Thiengo MOURA ${ }^{(2)}$ \\ Diego SANTANA ${ }^{(2)}$ \\ Brunella Ferrari Machado NASCIMENTO ${ }^{(2)}$ \\ Pierre Augusto Victor SILVA ${ }^{(3)}$
}

Recebido: 20 fev 2017

Revisado: 15 maio 2018

Aceito: 8 jul 2017

\section{Autor de} correspondência: Amanda Vargas Pereira amandavfono@gmail.com

Conflito de interesses: Os autores declaram não haver nenhum interesse profissional ou pessoal que possa gerar conflito de interesses em relação a este manuscrito.

\begin{abstract}
Resumo
O objetivo do presente estudo foi avaliar se o número de pacientes cadastrados corresponde ao número de acompanhados da população portadora de diabetes e hipertensão, do bairro Jardim Surubi no município de Itaperuna/RJ, no ano de 2015. Para este fim se utilizaram dados secundários da Estratégia de Saúde da Família - ESF do bairro Jardim Surubi no Município de Itaperuna/RJ no ano de 2015, extraídos Sistema de Informação da Atenção Básica - SIAB. A população alvo do estudo foram as 943 famílias cadastradas na Unidade de Saúde da Família. A coleta dos dados eletrônicos foi realizada entre os meses de janeiro a dezembro de 2015. A média de moradores do bairro Surubi, no ano de 2015 é de 3.076 pessoas. Em relação aos diabéticos 1.771 pacientes são cadastrados em todas as microáreas, destes cadastrados 1.486 são acompanhados e 285 estão sem acompanhamento. Dos hipertensos, 5.152 são pacientes cadastrados, sendo 4.329 acompanhados e 823 não estão acompanhados. Conclui-se que a cobertura de acompanhamentos pela equipe de saúde da família, está abaixo do desejado, reafirmando assim a necessidade do aumento de percentual de atendimento para esta população, colaborando para o controle e diminuição das prevalências.
\end{abstract}

Descritores: Hipertensão; Diabetes; Estratégia de Saúde da Família. 


\begin{abstract}
The objective of the present study was evaluated by the number of patients enrolled corresponding to the number of patients followed by the population with diabetes and hypertension, in the Jardim Surubi neighborhood in the city of Itaperuna, RJ, in the year 2015. This is a cross-sectional study with an Approach Quantitative. For this purpose, secondary data from the Family Health Strategy (FSE) of Bairro Jardim Surubi in the Municipality of Itaperuna / RJ were used in the year 2015, extracted the Basic Attention Information System (SIAB). The study population was the 943 families enrolled in the Family Health Unit. The electronic data collection was between January and 2015. The average number of residents in the Surubi neighborhood in the year 2015 is 3,076 people. Regarding diabetics, 1,771 patients are enrolled in all microdata, these 1,486 enrollees are followed up and 285 are unmonitored. Of the hypertensive patients, 5,152 are registered patients, of whom 4,329 are followed up and 823 are unaccompanied. It is concluded that a follow-up coverage by the family health team is lower than desired, thus reaffirming the need to increase the cost of care for this population, collaborating to control and reducing the prevalence of these pathologies.
\end{abstract}

Keywords: Hypertension; Diabetes; Family Health Strategy.

\title{
Resumen
}

El objetivo del presente estudio fue evaluar si el número de pacientes catastros corresponde al número de acompañados de la población portadora de diabetes e hipertensión del barrio Jardim Surubi en el municipio de Itaperuna / RJ, en el año 2015. Para este fin se utilizaron datos secundarios de la enfermedad Estrategias de Salud de la Familia (ESF) del barrio Jardim Surubi en el Municipio de Itaperuna / RJ en el año 2015, extraídos Sistema de Información de la Atención Básica (SIAB). La población objetivo del estudio fueron las 943 familias registradas en la Unidad de Salud de la Familia. La recolección de los datos electrónicos se realizó entre los meses de enero a diciembre de 2015. El promedio de habitantes del barrio Surubi, en el año 2015 es de 3.076 personas. En cuanto a los diabéticos 1.771 pacientes son registrados en todas las microáreas, de estos registrados 1.486 son acompañados y 285 están sin acompañamiento. De los hipertensos, 5.152 son pacientes registrados, siendo 4.329 acompañados y 823 no están acompañados. Se concluye que la cobertura de acompañamientos por el equipo de salud de la familia, está por debajo de lo deseado, reafirmando así la necesidad del aumento de porcentaje de atención para esta población, colaborando para el control y disminución de las prevalencias de estas patologías.

Palabras-claves: Hipertensión; Diabetes; Estrategias de Salud de la Familia.

\section{Introdução}

Nas últimas décadas observa-se uma mudança no perfil das doenças na população, em decorrência da transição epidemiológica, demográfica e nutricional. A transição epidemiológica pode ser definida como mudança do perfil de morbimortalidade, caracterizando-se pela redução das doenças infectocontagiosas e aumento das doenças crônicas não transmissíveis - DCNT. Diversos fatores são relevantes para estas mudanças 
como a urbanização, acesso a serviços de saúde e meios diagnósticos, melhoria nas condições de vida e saúde, redução da mortalidade e aumento da expectativa de vida. ${ }^{1}$

As DCNT compreendem prioritariamente a um grupo de doenças cardiovasculares, câncer, diabetes e doenças respiratórias crônicas. São doenças que geralmente estão relacionadas a causas múltiplas, de início gradual e com duração incerta. Apresentam, clinicamente, períodos de agudização e melhora do quadro, podendo ocasionar lesões incapacitantes. ${ }^{2,3}$

A estimativa de morte por DCNT é elevada em países de baixa e média renda, em comparação com países ricos. Quase dois terços de mortes prematuras em adultos nas idades entre 15 e 69 anos estão relacionados a estas condições, e três quartos das mortes adultas são relacionadas a estas circunstâncias. ${ }^{4}$

Ao se analisarem o total de óbitos ocorridos no mundo, em 2008, 63\% deles foram em decorrência das DCNT. No Brasil, as DCNT também constituem a maior causa dos óbitos, representando $72,4 \%$ do total. ${ }^{5}$

Um controle adequado do metabolismo, assim como a adoção de medidas preventivas e curativas é em grande parte simples e capaz de prevenir ou retardar o aparecimento das complicações crônicas do diabetes mellitus, proporcionando uma melhor qualidade de vida ao paciente diabético. Da mesma forma, o controle da hipertensão arterial visa a diminuição de dano aos órgãos-alvo. Para o controle de ambas as doenças, são necessárias ações que abrangem mudanças no estilo de vida do indivíduo. ${ }^{6}$

O manejo do diabetes mellitus e da hipertensão arterial deve ser realizado dentro de um sistema hierarquizado de saúde, sendo sua base o nível primário de atendimento. A equipe de Saúde da Família possui um papel imprescindível neste processo, fazendo o levantamento epidemiológico. ${ }^{6}$

Diante destes fatos, o presente estudo se faz necessário, pois o conhecimento do perfil dos hipertensos e diabéticos pode auxiliar no desenvolvimento de posteriores medidas de adesão e conscientização, prevenção e promoção da saúde, assim como mudanças no 
estilo de vida. A informação é um relevante instrumento para gestores e planejadores de saúde na elaboração e avaliação de políticas públicas. Este conhecimento pode contribuir para transformar o retrato epidemiológico de Itaperuna, cidade situada no interior do Rio de Janeiro com uma área territorial de 1.105,341 Km2, possuindo uma população geral de 99.504 mil. $^{7}$

O objetivo do presente estudo foi avaliar se o número de pacientes cadastros corresponde ao número de acompanhados da população portadora de diabetes e hipertensão, do bairro Jardim Surubi no município de Itaperuna/RJ, no ano de 2015, cadastrados no Sistema de Informação da Atenção Básica - SIAB.

\section{Metodologia}

No presente estudo foram utilizados dados secundários extraídos do SIAB do Departamento de Informação e Informática do SUS - DATASUS do Município de Itaperuna que tem aproximadamente 99.504 mil habitantes, segundo Instituto Brasileiro de Geografia e Estatística - IBGE.

1. A população alvo do estudo foram usuários cadastrados na Unidade de Saúde da Família do bairro Jardim Surubi no Município de Itaperuna/RJ e cadastrados no SIAB do Município no ano de 2015, totalizando 5.152 hipertensos e 1.771 diabéticos cadastros. A coleta dos dados eletrônicos foi realizada entre os meses de janeiro a dezembro de 2015.

2. Foram consideradas as seguintes variáveis: microáreas $(01,05,09,14,15,51,54$ e 72, e número de usuários diabéticos e hipertensos cadastrados e acompanhados por cada microárea. 
3. Os dados foram inseridos em planilhas do programa Microsoft Office Exce/ 2016 e estão representados em gráficos por pirâmide gerados pelo programa GraphPad Prism 7.0.

\section{Resultados}

A média de moradores do bairro Surubi, no ano de 2015 foi de 3.076 pessoas, sendo que a, Unidade de Saúde da Família deste bairro possui 08 microáreas, sendo estas numeradas como microáreas 01, 05, 09, 14, 15, 51, 54 e 72, totalizando 943 famílias cadastradas. Dessas, a micro área 01 é responsável por 47 famílias, a micro área 05 por 161 famílias, a micro área 09 por 154 famílias, a micro área 14 por 155 famílias, a micro área 15 por 132 famílias, a micro área 51 por 53 famílias, a micro área 54 por 150 famílias e a micro área 72 por 91 famílias. $^{8}$

De acordo com a Figura 1 pode-se verificar o índice de diabéticos e hipertensos cadastrados e acompanhados. Em relação aos diabéticos 1.771 pacientes são cadastrados em todas as microáreas, destes cadastrados 1.486 são acompanhados e 285 estão sem acompanhamento. Dos hipertensos, 5.152 são pacientes cadastrados, sendo 4.329 acompanhados e 823 não estão acompanhados.

Estes dados evidenciam que a ausência de acompanhamentos de ambas as patologias pode levar a sérios agravos de saúde, além de evidenciar um aumento do número de internações hospitalares e alto custo nos tratamentos. ${ }^{6}$

Diante disso, é possível observar que existem diferentes níveis de cobertura pelas equipes de Programa Saúde da Família - PSF (Figuras 2 e 3). Notar essa situação é de grande relevância, visto que, uma maior ou menor cobertura pode representar diferenças marcantes no volume de cadastros, assim como no nível de acompanhamento pelas equipes de saúde 6 , bem como a redução ou prevenção de DCNT podendo também reduzir o quadro de Acidente Vascular Cerebral - AVC.

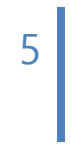


ISSN 2179-6750

\section{Pirâmide da População Total}

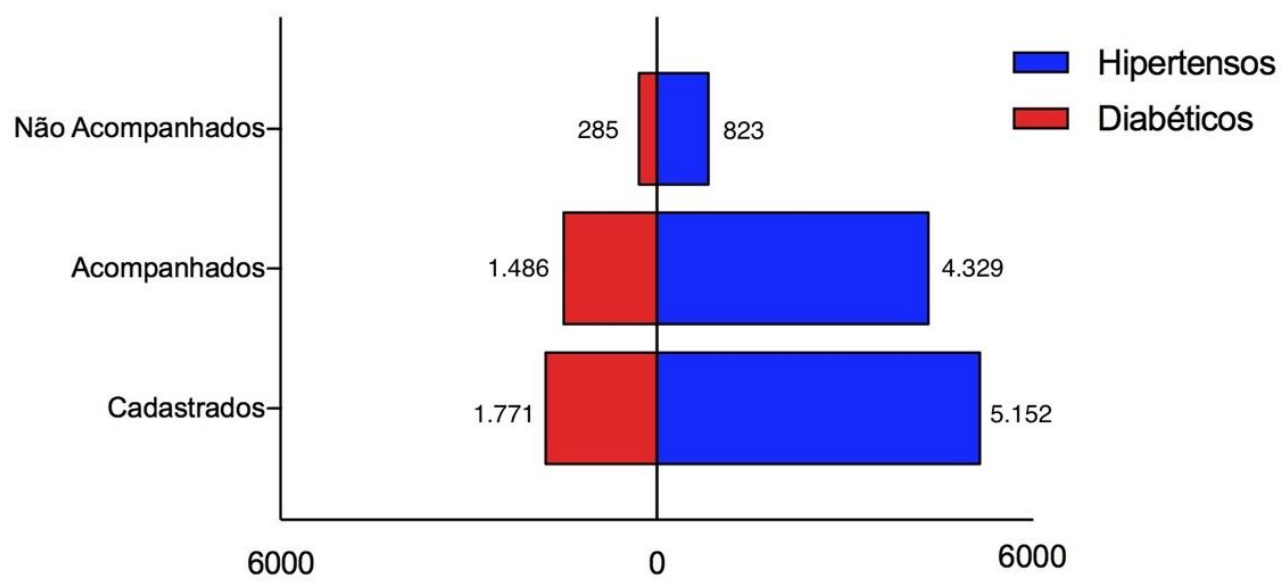

Figura 1. Gráfico acima apresenta o número de diabéticos e hipertensos cadastrados, acompanhados e não acompanhados na população do Jardim Surubi. Podemos observar que o número de hipertensos em relação aos diabéticos é maior, o que nos aponta para um perfil de população hipertensa.

Fonte: Elaboração própria (2017).

Pirâmide da População Diabética Anual por Microárea

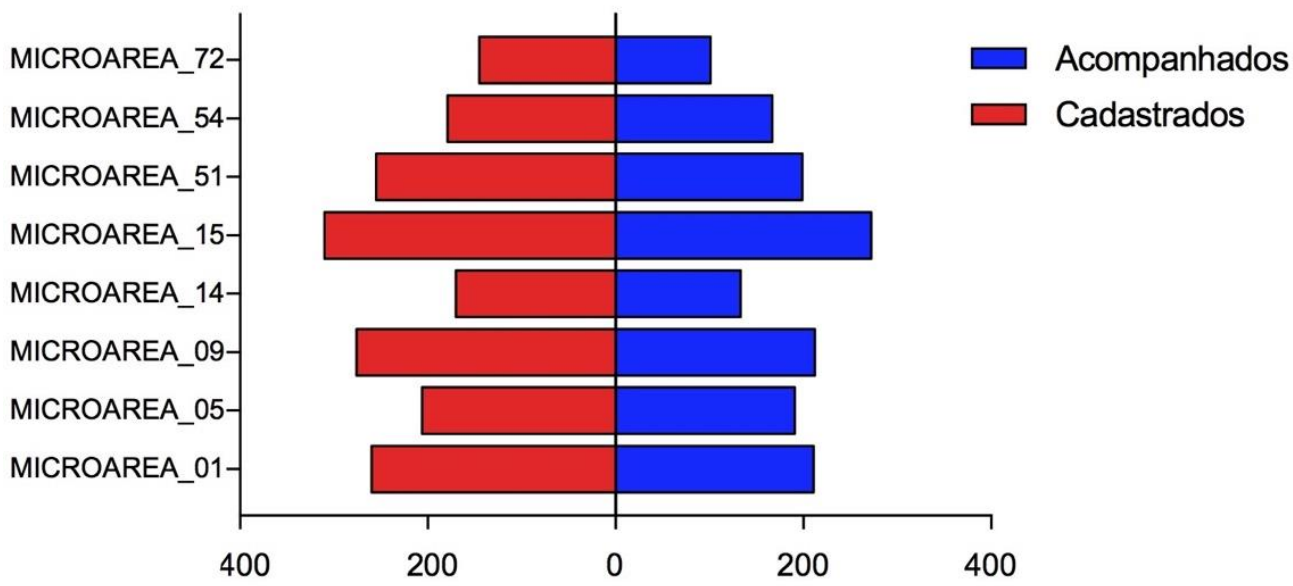

Figura 2. Gráfico acima apresenta o número de diabéticos cadastrados e acompanhados por microárea na população do Jardim Surubi. Onde podemos observar que nem todos os diabéticos cadastrados, estão sendo acompanhados.

Fonte: Elaboração própria (2017). 
Sabe-se que é de fundamental importância que os profissionais de saúde das Unidades Básicas de Saúde, conheçam bem o território de abrangência, assim como a população adstrita, pois é através de conhecimentos que é possível planejar ações direcionadas a real necessidade daquela população. Ao analisar a variação de famílias cadastradas, acompanhadas e não acompanhadas por microárea é possível constatar que há uma sobrecarga para alguns profissionais, neste caso, os agentes comunitários de saúde e isso interfere na qualidade do serviço prestado, podendo ocasionar uma diminuição no acompanhamento dos pacientes e, consequentemente, uma menor assistência. ${ }^{9}$

\section{Pirâmide da População Hipertensa Anual por Microárea}

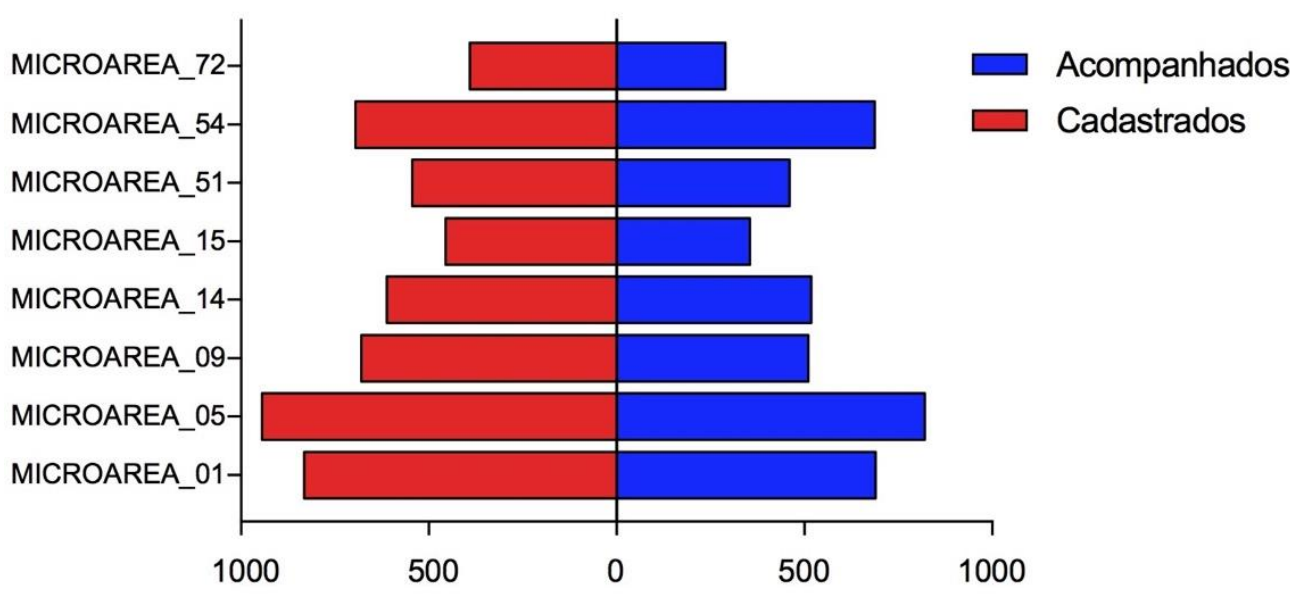

Figura 3. Gráfico acima apresenta o número de hipertensos cadastrados e acompanhados na população do Jardim Surubi divididos por microárea, observamos também que em todas as microáreas a uma grande população de hipertensos cadastrada que não recebem o acompanhamento.

Fonte: Elaboração própria (2017).

Os resultados demonstram a necessidade de um maior investimento na saúde local do bairro Jardim Surubi, visto que, 285 pacientes diabéticos e 823 pacientes hipertensos não recebem acompanhamento, podendo assim ocorrer o abandono ou baixa adesão ao tratamento. Quando não há um acompanhamento correto e o paciente hipertenso não segue o tratamento prescrito pelo médico aumenta-se o risco para que o paciente 
desenvolva um acidente vascular encefálico ou insuficiência cardíaca por exemplo. O diabetes quando não tratado corretamente pode ocasionar amputações e cegueira. ${ }^{11,12,13}$

Ao analisar a microárea 54 podemos observar que quase todos seus pacientes cadastrados recebem o acompanhamento pela Unidade Básica de Saúde - UBS evidenciando assim, a necessidade de investimento para que alcance todos os pacientes cadastrados de maneira adequada. Essa falta de acompanhamento que ocorre não só no Brasil é alarmante, pois o diabetes e a hipertensão são doenças que levam ao desenvolvimento de outras mais. 8,10

\section{Discussão}

Os resultados obtidos nessa pesquisa demonstram que dos usuários cadastrados na Unidade de Saúde Jardim Surubi, existe uma predominância de hipertensos em relação ao número de pacientes diabéticos. No Brasil, segundo dados epidemiológicos demonstrados pela pesquisa denominada Vigitel Brasil, a hipertensão arterial atinge $22,7 \%$ da população adulta brasileira e a incidência de hipertensos, no Estado do Rio de Janeiro, foi de $24 \%$ e a incidência de pacientes diabéticos foi de 7\%, corroborando assim com os resultados da presente pesquisa onde há um predomínio da hipertensão arterial sobre a diabetes. ${ }^{20,21}$

Ainda de acordo com a pesquisa Vigitel Brasil, a incidência da hipertensão arterial se eleva com o decorrer dos anos. Se entre 18 e 24 anos, somente 5,4\% da população afirmou ter recebido diagnóstico de hipertensão, aos 55 anos essa proporção é dez vezes maior, abrangendo acima da metade da população (50,5\%). A partir dos 65 anos, a hipertensão passa a ser observada em 59,7\% dos brasileiros, o que não foi possível analisar em nossos estudos por não possuir acessos as variáveis como idade. ${ }^{20,21}$

Estudos epidemiológicos têm verificado não somente a elevação da predominância da hipertensão arterial com a idade, mas também a sua incidência associada a outros fatores de risco como obesidade, estilo de vida e metabólicos. ${ }^{22}$ 
Os elevados índices de hipertensão e diabetes encontrados na Unidade de Saúde Jardim Surubi, localizada em Itaperuna, refletem o atual cenário brasileiro em que houve redução na taxa de crescimento populacional, com um aumento significativo no número de idosos. A população de Itaperuna, segundo o Censo de 2010, era de 95.841 mil habitantes e a estimativa de 2016 é de 99.504 mil habitantes. Entre 2000 e 2010 a taxa média de crescimento anual foi de 1,01\%, sendo que, entre 1991 e 2000 , essa mesma taxa foi de $1,18 \%$, demonstrando assim, uma redução na taxa de crescimento da população. ${ }^{14}$

Com essa diminuição do crescimento populacional e aumento do número de idosos, houve uma conversão do perfil epidemiológico, ocorrendo um predomínio da morbidade e mortalidade pelas doenças crônicas não transmissíveis e não mais pelas doenças infecto contagiosas, como eram em décadas passadas. Das DCNT, quatro grupos se destacam: cardiovasculares, como por exemplo a hipertensão arterial, câncer, doença respiratória crônica e diabetes, sendo a hipertensão e a diabetes os de maior prevalência. ${ }^{3}$

Diante disso, o Ministério da Saúde implantou, em 2001, o Plano de Reorganização da Atenção aos portadores de Hipertensão Arterial Sistêmica (HAS) e Diabetes Mellitus (DM), enfatizando a rede de Atenção Primária à Saúde (APS). Esse trabalho foi resultado de uma parceria entre as Sociedades Científicas de Hipertensão Arterial, Cardiologia, Nefrologia, Diabetes, Endocrinologia e Metabologia. ${ }^{15}$

Com o intuito de reorganizar os serviços, promovendo uma assistência de qualidade aos hipertensos e diabéticos foi destacado, por este plano, questões como a confirmação de casos suspeitos, a elaboração de protocolos clínicos, treinamentos dos profissionais de saúde, além da criação de um sistema informatizado de cadastramento e acompanhamento de hipertensos e diabéticos denominado Sis-HIPERDIA, mais conhecido como HIPERDIA. ${ }^{15}$

O HIPERDIA foi criado com o objetivo de monitorar os pacientes cadastrados, assim como gerar informações a respeito da aquisição, dispensação e distribuição dos medicamentos para os pacientes inseridos nesse programa. Este sistema é composto pelos subsistemas Municipal e Federal, sendo que os bancos de dados alimentados pelas 
Secretarias Municipais de Saúde (SMS), fazem parte de uma base nacional de informações, que pode ser acessada através internet, por meio de um site específico do DATASUS, órgão este do Ministério da Saúde e que é responsável pelos sistemas informatizados. ${ }^{15}$

Para constituição das informações faz-se necessário o preenchimento da Ficha de Cadastro do paciente hipertenso e/ou diabético pelos profissionais de saúde. Os dados são digitados, proporcionando dados sobre quantos são os pacientes portadores de diabetes mellitus (DM), portadores de hipertensão arterial (HA) e quantos deles são acompanhados. ${ }^{15}$ Para que ocorra manutenção do sistema, deve-se informar as datas de óbitos dos pacientes cadastrados, comprovadas por atestados. Pacientes que mudam de Unidade Básica de Saúde também precisam ter seus cadastros desativados dessas unidades e refeitos na nova Unidade de assistência. Dessa forma, é possível obter um sistema com dados fidedignos e, assim, promover ações direcionadas ao perfil de morbidade e mortalidade das microáreas atendidas, assim como promover ações de promoção e prevenção. ${ }^{15}$

Ainda assim, perdura-se uma porção não diagnosticada de casos de hipertensão e diabetes, que tende a diminuir com o aumento do acesso aos serviços de saúde e com a organização da atenção básica. Conhecer essa porção, aumenta a relevância da avaliação de programas e estratégias que realizem busca ativa e rastreamento da população para essas doenças. $^{21}$

Baseados nos resultados encontrados, em que é possível observar o elevado número de pacientes hipertensos e diabéticos, assim como o negativo impacto socioeconômico gerado por essas doenças, a Hipertensão Arterial Sistêmica e o Diabetes Mellitus têm se mostrado como um dos grandes desafios do século XXI e que demandam intervenções de prevenção e promoção da saúde, assim como mudanças no estilo de vida sendo, portanto, ações não somente proporcionadas pelos órgãos de saúde, mas que dependem também do governo e da sociedade ${ }^{5}$. Ao preconizar intervenções direcionadas ao enfrentamento das DCNT, a Organização Mundial de Saúde (OMS) teve como foco quatro principais fatores de risco: tabagismo, inatividade física, obesidade e consumo excessivo de álcool. O tabagismo 
responde por $71 \%$ dos casos de câncer de pulmão, além de estar envolvido também com complicações cardiovasculares. A inatividade física, que está intimamente ligada a obesidade, aumenta em 20 a 30\% a chance de mortalidade. Além desses fatores, 25\% da população adulta relata ter tido algum problema, seja social ou físico, devido a ingestão elevada de álcool. ${ }^{5}$

O processo de enfrentamento das DCNT baseia-se nas suas causas, nos quatro principais fatores de risco e nas medidas preventivas. A vigilância das doenças crônicas pode ser elucidada como um conjunto de ações com o intuito de conhecer a distribuição, carga e a tendência dessas doenças, para assim promover ações direcionadas e efetivas. No Brasil, a vigilância é feita através de inquéritos realizados de forma periódica, onde são monitorados os fatores de risco. E para isso, dados socioeconômicos, demográficos e comportamentais são coletados. ${ }^{16}$

Outro instrumento utilizado no enfrentamento das DCNT é a Epidemiologia, que é um dos ramos da Medicina de grande relevância no desafio das doenças crônicas. Na Epidemiologia, enfatizam-se estratégias preventivas precoces que proporcionam, por exemplo, nutrição adequada desde a infância, evitando assim, possíveis complicações cardiovasculares e obesidade. Além desses mecanismos, a perspectiva ecossocial explica como as relações sociais, políticas e econômicas nas quais o indivíduo está inserido, ou seja, os determinantes sociais de saúde, influenciam nas suas condições de saúde. Essa perspectiva reconhece que o processo de adoecimento vai além dos fatores biológicos, mas envolve também as iniquidades sociais. ${ }^{5}$

A realização do cuidado integral das doenças crônicas não transmissíveis deve ocorrer com o fortalecimento do sistema de vigilância em saúde que estão entre as diretrizes e recomendações do Ministério da Saúde. Dessa forma, é imprescindível agrupar dados sobre a situação de saúde da população, tendo em vista elucidar as vulnerabilidades e promover um maior ajuste das estratégias de informação. ${ }^{17}$ 
Além disso, outra questão de grande importância para o confronto às doenças crônicas é a promoção da saúde. A ação de promover saúde tem se mostrado progressivamente mais presente no cotidiano dos profissionais de saúde e, principalmente, na prestação de serviços na área de respeito de promoção à saúde através de Leavell e Clark, em 1976, utilizando o modelo da história natural da doença e dividindo as ações de prevenção em três níveis: o primário, secundário e terciário. O conceito de promoção a saúde foi se modernizando por meio das conferências mundiais de saúde como por exemplo: Ottawa, Adelaide e Jakarta. ${ }^{8}$

Suas ações são voltadas tanto a grupos sociais como a indivíduos, através de políticas públicas direcionadas ao ambiente físico, social, político, econômico, cultural e no apoio da comunidade. Diante disso, pode-se compreender que os mecanismos de promoção à saúde podem proporcionar mudanças no estilo de vida, bem como as condições sociais, econômicas e ambientais que influenciam saúde, promovendo uma maior equidade. ${ }^{18}$

O Programa de Saúde da Família - PSF surgiu como ferramenta para romper o modelo hegemônico assistencialista, curativista e hospitalocêntrico. Para isso, ele institui que o paciente seja atendido de forma integral e as ações realizadas sejam de promoção à saúde e prevenção de doenças. Diante disso, a equipe da saúde da família mostra-se como peça fundamental para implantação e efetividade dessas ações, devido à proximidade da equipe no cotidiano das famílias atendidas pela Unidade Básica de Saúde - UBS. As ações promovidas pela equipe multiprofissional, no âmbito da Saúde da Família, abrangem visitas domiciliares, ações educativas e atendimentos no consultório. ${ }^{18}$

A gestão do PSF depende da magnitude da população que é beneficiada das ações e serviços de saúde. Além disso, quando se enfatiza no campo da avaliação a importância dos estudos de cobertura uma temática que não tem sido priorizada, isso colabora para o aperfeiçoamento de estratégias de estudo para essa etapa fundamental de monitoramento. ${ }^{19}$ 
Além disso, é fundamental a educação permanente da equipe multiprofissional, independente se ela atua na assistência ou no planejamento das ações. Portanto, pode-se concluir que o PSF tem se mostrado de grande importância para a consolidação das ações de promoção, prevenção e por encontrar-se disposto a novas propostas com o objetivo de melhorar a assistência as comunidades. ${ }^{18}$

\section{Conclusão}

Conclui-se que a cobertura de acompanhamentos pela equipe de saúde da família, está abaixo do desejado, reafirmando assim a necessidade do aumento de percentual de atendimento para esta população, colaborando para o controle e diminuição das prevalências destas patologias.

Neste sentido, este estudo traz elementos para o debate em torno de novas estratégias para o controle e diminuição das DCNT priorizando a prevenção e o tratamento precoce.

\section{Referências}

1. Duarte BM. Fatores de risco e proteção para doenças crônicas não transmissíveis na população de Belo Horizonte [dissertação]. Belo Horizonte: UFMG; 2010. 105 f. Disponível em: http://www.bibliotecadigital.ufmg.br/dspace/handle/1843/GCPA84RH8W.

1. Achutti A; Azambuja MIR. Doenças crônicas não-transmissíveis no Brasil: repercussões do modelo de atenção à saúde sobre a seguridade social. Cien Saude Colet. 2004;9(4):833-40. http://dx.doi.org/10.1590/S1413-81232004000400002. 
3. Brasil. Ministério da Saúde. Diretrizes para o cuidado das pessoas com doenças crônicas nas redes de atenção à saúde e nas linhas de cuidado prioritárias. Brasília, DF: MS; 2013 [acesso 9 out 2016]. Disponível em:

http://bvsms.saude.gov.br/bvs/publicacoes/diretrizes\%20 cuidado pessoas $\% 20$ doenca s cronicas.pdf.

4. Goulart FAA. Doenças crônicas não transmissíveis: estratégias de controle e desafios e para os sistemas de saúde. Brasília, DF; 2011 [acesso em 17 ago 2016]. Disponível em: http://apsredes.org/site2012/wp-content/uploads/2012/06/CondicoesCronicas flavio1.pdf.

5. Duncan BB, Chor D, Aquino EML, Bensenor IM, Schimidt MI, Lotufo PA, et al. Doenças crônicas não transmissíveis no Brasil: prioridade para enfrentamento e investigação. Rev Saude Publica 2012;46(Supl):126-34. http://dx.doi.org/10.1590/S003489102012000700017.

6. Malfatti CRM, Assunção AN. Hipertensão arterial e diabetes na Estratégia de Saúde da Família: uma análise da frequência de acompanhamento pelas equipes de Saúde da Família. Cienc Saude Colet. 2011 [acesso em 09/10/2016];16(sup 1):1383-8. http://dx.doi.org/10.1590/S1413-81232011000700073.

7. Instituto Brasileiro de Geografia e Estatística - IBGE [Internet]. [Rio de Janeiro]: IBGE; [s.d.] [Acesso em 09/10/2016]. Disponível em:

http://www.cidades.ibge.gov.br/xtras/perfil.php?lang $=\&$ codmun $=330220 \&$ search $=$ rio de-janeirolitaperuna.

8. Ministério da Saúde. SIAB - manual do sistema de informação de atenção básica. Brasília, DF: MS; 2016.

9. Figueiredo VL. Trabalho em equipe: um desafio para a equipe de saúde da família. Belo Horizonte, BH: 2012 [acesso em 12 out 2016]. Disponível em: https://www.nescon.medicina.ufmg.br/biblioteca/imagem/4038.pdf.

10. López-Jaramillo P, Sánchez RA, Diaz M, Cobos L, Bryce A, Parra-Carrillo JZ, et al. Consenso latino-americano de hipertensão em pacientes com diabetes tipo 2 e síndrome metabólica. Arq Bras Endocrinol Metab. 2014;28(3):205-25. http://dx.doi.org/10.1590/0004-2730000003019.

11. Brasil. Ministério da Saúde. V Diretrizes Brasileiras de Hipertensão Arterial. São Paulo: MS;2006 [acesso em 12 out 2016]. Disponível em: http://publicacoes.cardiol.br/consenso/2006/VDiretriz-HA.asp. 
12. Sociedade Brasileira de Diabetes. Diabetes. São Paulo: SBD; [s.d.] [acesso em 12/10/2016]. Disponível em: http://www.diabetes.org.br.

13. Paiva DCP, Bersusa AAS, Escuder MML. Avaliação da assistência ao paciente com diabetes e/ou hipertensão pelo Programa Saúde da Família do Município de Francisco Morato, São Paulo, Brasil. Cad Saude Publica. 2006;22(2):377-85. http://dx.doi.org/10.1590/S0102-311X2006000200015.

14. Itaperuna (RJ). Secretaria Municipal de Saúde. Plano Municipal de Saúde - 2014 a 2017. Itaperuna: SMS; 2013.

15. Chazan AC, Perez EA. Avaliação da implementação do sistema informatizado de cadastramento e acompanhamento de hipertensos e diabéticos (Hiperdia) nos municípios do estado do Rio de Janeiro. Rev APS. 2008 [acesso em 9 out 2016];11(1). Disponível em: https://aps.ufjf.emnuvens.com.br/aps/article/viewFile/197/80.

16. Brasil. Ministério da Saúde. Diretrizes e recomendações para o cuidado integral de doenças crônicas não-transmissíveis. Promoção da Saúde, Vigilância, Prevenção e Assistência. Brasília, DF: MS; 2008 [acesso em 9 out 2016]. Disponível em: http://bvsms.saude.gov.br/bvs/publicacoes/diretrizes\%20 cuidado pessoas\%20 doenca s cronicas.pdf.

17. Correia LOS, Padilha BM, Vasconcelos SML. Completitude dos dados de cadastro de portadores de hipertensão arterial e diabetes mellitus registrados no Sistema Hiperdia em um estado do Nordeste do Brasil. Cienc Saude Colet. 2014;19(9):1685-97. http://dx.doi.org/10.1590/1413-81232014196.02842013.

18. Noronha MGRCS, Cardoso DS, Moraes TNP, Centra ML. Resiliência: nova perspectiva na Promoção da Saúde da Família?. Cienc Saude Publica. 2009;14(2):497-506. http://dx.doi.org/10.1590/S1413-81232009000200018.

19. Aquino R, Barreto M.L. Programa Saúde da Família: acerca da adequação do uso do seu indicador de cobertura. Cad Saude Publica. 2008; 24(4):905-14. http://dx.doi.org/10.1590/S0102-311X2008000400022.

20. Brasil. Ministério da Saúde. Vigitel Brasil 2015 Saúde Suplementar: vigilância de fatores de risco e proteção para doenças crônicas por inquérito telefônico. Brasília, DF: MS; 2015 [acesso em 9 out 2016]. Disponível em:

http://www.ans.gov.br/images/stories/Materiais_para_pesquisa/Materiais_por_assunto/2 015_vigitel.pdf. 
21. Schimidt MI, Duncan BB, Hoffmann JF, Moura L, Malta DC, Carvalho RMSVI. Prevalência de diabetes e hipertensão no Brasil baseada em inquérito de morbidade auto-referida, Brasil, 2006. Rev Saude Publica, 2009;43(suppl 2):74-82. http://dx.doi.org/10.1590/S0034-89102009000900010.

22. Passos VMA, Assis TD, Barreto SM. Hipertensão arterial no Brasil: estimativa de prevalência a partir de estudos de base populacional. Epidemiol Serv Saude. 2006;15(1):35-45. http://dx.doi.org/10.5123/S1679-49742006000100003.

\section{Minicurrículo}

Amanda Vargas Pereira | ORCiD: 0000-0002-5953-3712

Fonoaudiologa. Mestrado em Doenças Infecciosas e Parasitárias pela Universidade Federal do Rio de Janeiro - UFRJ.

Amanda Thiengo Moura | ORCiD: 0000-0002-5908-4283

Graduanda em Medicina, UniRedentor.

Diego Santana | ORCiD: 0000-0001-8262-6661

Graduando em Medicina, UniRedentor.

Brunella Ferrari Machado Nascimento | ORCiD: 0000-0001-8585-4977

Graduanda em Medicina, UniRedentor.

Pierre Augusto Silva | ORCiD: 0000-0002-6367-9482

Fisioterapeuta. Coordenador de Graduação em Fisioterapia, UniRedentor. Mestrando em Ciências da Reabilitação e Desempenho Físico-Funcional, na Universidade Federal de Juiz de Fora - UFJF. 